\title{
Lattice Boltzmann method for solving non-equilibrium radiative transport problems
}

\author{
Abhinav Gairola, Hitesh Bindra* \\ Department of Mechanical and Nuclear Engineering, Kansas State University, Manhattan, \\ Kansas, 66506
}

\begin{abstract}
Recently developed lattice Boltzmann equation (LBE) framework $[1,2]$ for radiation transport is extended to solve time-dependent non-equilibrium radiative and neutron tranport problems. Dynamics of radiation and material energy exchange is modeled by coupling radiation transport equation with the material energy equation in a one-dimensional isotropically scattering homogenous medium. The LBE solutions are compared against the existing semi-analytical $P_{1}$ benchmark and show good agreement for different times. Furthermore, a two-dimensional $D_{2} Q_{8}$ lattice Boltzmann method is proposed for solving the neutron transport equation in a heterogenous media (e.g., checkerboard lattice with pure scattering and absorbing cells). The results obtained with LBE are in good agreement with the existing discrete ordinate method results for the benchmark problem.
\end{abstract}

\section{Keywords:}

Lattice Boltzmann, Radiation Transport, Non-equilibriuam, Radiation-Material interactions

\footnotetext{
*Corresponding author

Email address: hbindra@ksu . edu (Hitesh Bindra)
} 


\section{Nomenclature}

$\begin{array}{llll}\Omega & \text { Angular direction } & r & \text { Spatial location } \\ \alpha & \text { Radiative loss coefficient } & S & \text { Energy source } \\ \phi_{0} & \text { Total radiative flux } & s_{0} & \text { Neutron source } \\ \psi & \begin{array}{l}\text { Non dimensional radiation in- } \\ \text { tensity }\end{array} & T & \text { Temperature } \\ & \text { Absorption cross-section } & t & \text { Time } \\ \sigma_{a} & T_{H} & \text { Reference temperature } \\ \sigma_{s} & \text { Scattering cross-section } & v & \text { Speed of radiative transport } \\ \sigma_{t} & \text { Total cross-section } & W_{i} & \text { Directional weights } \\ \theta & \text { Non dimensional temperature } & I & \text { Radiation intensity } \\ C_{v} & \text { Specific heat } & & \end{array}$

\section{Introduction}

Most of the leading computer codes for solving radiation and neutron transport physics are based on probabilistic Monte Carlo methods [3, 4] (e.g., MCNP, KENO) or the deterministic discrete ordinate methods $\left(\mathrm{DOM}-S_{N}\right.$ ) (e.g., Denovo [5], DeCART [6]) or methods of characteristics (MOC). In Monte Carlo codes, the computational domain is composed of an assembly of several primitive geometries (e.g., cubes, cylinders, spheres), and particle populations are sampled and tracked in those subdomains for their probabilistic interactions with matter. Whereas, the deterministic methods for radiation transport usually rely on a spatial mesh with angular phase space and energy discretizations. Although these highly specialized discretizations are extremely useful for transport only applications, they are very difficult to use in a multi-physics setup for coupling to fluid flow (single and two 
phase laminar or turbulent flows), conjugate heat transfer, and structural mechanics continuum codes. One reason being the entirely different spatial and temporal discretization and convergence approaches (e.g., finite volume, finite element and finite difference methods on finely-resolved unstructured tetrahedral, hexahedral or polyhedral grids) used in the continuum mechanics codes, which makes the grid transformation and interpolated field mapping very cumbersome and may even lead to a loss of accuracy. Therefore, there is a clear need to explore new methods that can allow tightly coupled solutions of both the transport and continuum physics in the same spatial and temporal discretization framework. The lattice Boltzmann equation (LBE) method is a relatively new mesoscopic kinetic approach that can provide such a single solver framework. Recently, a LBE approach was developed [1] to solve the steady state radiation transport problems in one and two dimensions. Results for a $D_{2} Q_{4}$ lattice-equivalent to discrete ordinates $S_{2}$ method for time-independent problems - were presented in that work [1]. In this paper, we further extend the LBE approach to solve nonequilibrium radiation and neutron transport problems in one and two dimensions and demonstrate agreements with the existing numerical benchmarks.

Nonequilibrium radiation transport with coupled dynamic material properties has always been a problem of significant importance for astrophysics, solar energy, nuclear engineering, and radiology applications. This coupled problem requires the solution to the linear Boltzmann transport equation in conjunction with linear or nonlinear equations for material dynamics. Consequently most of the practically significant cases do not have analytical solutions and specialized numerical methods are therefore required to solve them. However, for some one-dimensional problems, analytical and semi-analytical $P_{n}$ solutions do exist which can be used 
for validating the numerical methods. One such classical problem in radiationmaterial energy exchange is known as the Marshak wave problem. The general form of that equation is nonlinear due to the temperature term [7] and have to be linearized [8] to produce analytical, $P_{n}$ and diffusion-approximation based solutions. Ganapol and Pomraning [9] have solved a particular nonequilibrium radiative transfer problem in which a free surface is irradiated with a constant radiation flux. They used a method of multiple collisions and provided solution in the form of an infinite series of integrals. Su and Olson [10] later developed a complete analytical solution in one dimension to a nonequilibrium transport problem with a source term.

In this paper, we propose an extended LBE to solve the coupled nonequilibrium problem in one-dimension and compare $D_{1} Q_{2}$ LBE results with the analytical $P_{1}$ results from McClarren et al. [11]. The same LBE approach is then shown to be extensible for problems in two dimensions by solving time dependent transport problems on $D_{2} Q_{8}$ and $D_{2} Q_{16}$ LBE lattices. The results are presented for a benchmark problem having a heterogenous checkerboard lattice of absorbing and scattering media. This two-dimensional checkerboard problem was also used by Brunner [12] as a test case to compare different radiation transport methods (diffusion, flux limited diffusion, $P_{1}, P_{7}, S_{6}$ and Implicit Monte Carlo). The following section describes the classical equations, assumptions, and approximations used in this paper to model the radiation transport physics with the LBE.

\section{Mathematical formulation}

The interaction of radiation and material energies is modeled by coupling the radiation transport equation (RTE) with the material energy balance equation. Assum- 
ing homogenous materials and isotropic scattering, the set of governing equations for non-equilibrium radiative transfer can be written as

$$
\begin{array}{r}
\frac{1}{v} \frac{\partial I(r, \Omega, t)}{\partial t}+\Omega . \nabla I(r, \Omega, t)=\sigma_{a}\left[\frac{1}{4 \pi} \alpha T(r, t)^{4}-I(r, \Omega, t)\right] \\
+\sigma_{s}\left[\frac{1}{4 \pi} \int_{4 \pi} I\left(r, \Omega^{\prime}, t\right) d \Omega^{\prime}-I(r, \Omega, t)\right]+S(r, \Omega, t)
\end{array}
$$

and

$$
\frac{1}{v} C_{v} \frac{\partial T(r, t)}{\partial t}=\sigma_{a}\left[\int_{4 \pi} I(r, \Omega, t) d \Omega-\alpha T(r, t)^{4}\right]
$$

where $I(r, \Omega, t)$ is the radiation intensity at any spatial location $r$ and time $t$ in the direction $\Omega ; v$ is the speed of radiative transport; $T(r, t)$ is the temperature; $\sigma_{a}$ is the absorption cross-section; $\sigma_{s}$ is the scattering cross-section; $S(r, \Omega, t)$ is the volumetric energy source; $C_{v}$ is the specific heat; and $\alpha$ is the radiative loss coefficient. These equations can also be written using total cross-section $\sigma_{t}=\sigma_{a}+\sigma_{s}$ as a parameter.

By assuming $\sigma_{a}$ and $\sigma_{s}$ to be constant [10] and $C_{v}$ to be proportional to the cube of temperature $T$, as modeled by Pomraning [8],

$$
C_{v}=\alpha T^{3}
$$

Eqs. (1) and (2) can be linearized. Following non-dimensionalization can be carried out with respect to $\alpha T_{H}{ }^{4}$, where $T_{H}$ is a reference temperature:

$$
\begin{aligned}
& \psi(r, \Omega, t)=\frac{I(r, \Omega, t)}{\alpha T_{H}{ }^{4}}, \\
& s(r, \Omega, t)=\frac{S(r, \Omega, t)}{\alpha T_{H}{ }^{4}},
\end{aligned}
$$




$$
\theta(r, t)=\frac{T(r, t)^{4}}{T_{H}{ }^{4}},
$$

and which results in the following set of governing equations:

$$
\begin{gathered}
\frac{1}{v} \frac{\partial \psi(r, \Omega, t)}{\partial t}+\Omega \cdot \nabla \psi(r, \Omega, t)=\sigma_{a}\left[\frac{1}{4 \pi} \theta(r, t)-\psi(r, \Omega, t)\right] \\
+\sigma_{s}\left[\frac{1}{4 \pi} \int_{4 \pi} \psi\left(r, \Omega^{\prime}, t\right) d \Omega^{\prime}-\psi(r, \Omega, t)\right]+s(r, \Omega, t), \\
\frac{1}{v} \frac{\partial \theta(r, t)}{\partial t}=\sigma_{a}\left[\int_{4 \pi} \psi\left(r, \Omega^{\prime}, t\right) d \Omega^{\prime}-\theta(r, t)\right] .
\end{gathered}
$$

After introducing a finite set of directions and associated angular distribution functions or radiation intensity for each lattice direction, above equations (7) and (8) can be discretized to yield the equivalent LBE, and are provided below:

$$
\begin{gathered}
\psi_{\Omega}\left(r+v_{\Omega} \Delta t, t+\Delta t\right)-\psi_{\Omega}(r, t)= \\
\sigma_{a}\left[W_{\Omega} \theta(r, t)-\psi_{\Omega}(r, t)\right] \Delta t \\
+\sigma_{s}\left[W_{\Omega} \sum_{\Omega^{\prime}} W_{\Omega^{\prime}} \psi_{\Omega^{\prime}}(r, t)-\psi_{\Omega}(r, t)\right] \Delta t \\
+s_{\Omega}(r, t) \Delta t \\
\theta(r, t+\Delta t)-\theta(r, t)=\sigma_{a}\left[\sum_{\Omega^{\prime}} W_{\Omega^{\prime}} \psi_{\Omega^{\prime}}(r, t)-\theta(r, t)\right] \Delta t .
\end{gathered}
$$

For a two dimensional Cartesian LBE lattice, equation (9) reduces to

$$
\begin{array}{r}
\psi_{i}\left(x+v_{i, x} \Delta t, y+v_{i, y} \Delta t, t+\Delta t\right)=\psi_{i}(x, y, t) \\
-\Delta t\left(\sigma_{t} \psi_{i}(x, y, t)+\sigma_{s} W_{i} \sum_{j} W_{j} \psi_{j}(x, y, t)+W_{i} s(x, y, t)\right)
\end{array}
$$


where $\psi_{i}(x, y, t)$ is the discrete angular neutron flux in the $i^{t h}$ lattice direction at $(x, y)$ location at time $t$. Lattice velocities $v_{i, x}$ and $v_{i, y}$ are chosen such that the distance traveled by particles in a discrete time interval $\Delta t$ i.e., $v_{i, x} \Delta t$ and $v_{i, y} \Delta t$ equals to the distance between the neighboring lattice points. Furthermore, $W_{i}$ represents directional weighting factor for each direction of travel.

While approximating the integrals in the equations (7) and (8) numerically, weights $W_{j}$ were set to ' 1 '.

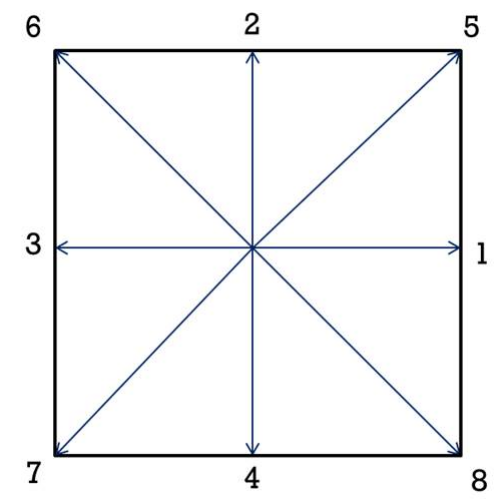

Figure 1: Lattice directions for two-dimensional $D_{2} Q_{8}$ system.

In 2D, the most commonly used lattice framework for LBE is $D_{2} Q_{8}$ typically used to solve fluid mechanics problems, which has 8 directions $=1-8$ (Fig. 1), and the standard corresponding notation for $v_{i}$ can be written as:

$$
v_{i}= \begin{cases}v\left(\cos \frac{(i-1) \pi}{2}, \sin \frac{(i-1) \pi}{2}\right) & i=1-4 \\ \sqrt{2} v\left(\cos \left(\frac{(i-5) \pi}{2}+\frac{\pi}{4}\right), \sin \left(\frac{(i-5) \pi}{2}+\frac{\pi}{4}\right)\right) & i=5-8\end{cases}
$$

The effective speed of the first four cardinal directions $i=1-4$ is equal to $v$ and for diagonal directions $i=5-8$ are equal to $\sqrt{2} v$. The weights $W_{i}$ for directions with the same speeds are equal because of symmetry. The weights for 
each cardinal and diagonal direction lead to isotropic generalized lattice tensors of rank 2 and 4 [13, 14]. This is achieved by conservation of lattice angular moments up to the fourth order over scattering or any other radiation interaction. In case of an isotropic function $w(\mu)$, the odd moments vanish,

$$
\begin{gathered}
\sum_{i=1}^{8} v_{i \alpha} W_{i}=\int_{-1}^{1} w(\mu) \mu d \mu=0 \\
\sum_{i=1}^{8} v_{i \alpha} v_{i \beta} v_{i \gamma} W_{i}=\int_{-1}^{1} w(\mu) \mu_{\alpha} \mu_{\beta} \mu_{\gamma} d \mu=0,
\end{gathered}
$$

and the even moments can be expressed as

$$
\begin{gathered}
\sum_{i=1}^{8} W_{i}=\int_{-1}^{1} w(\mu) d \mu=1 \\
\sum_{i=1}^{8} v_{i \alpha} v_{i \beta} W_{i}=\int_{-1}^{1} w(\mu) \mu_{\alpha} \mu_{\beta} d \mu=\delta_{\alpha \beta} \\
\sum_{i=1}^{8} v_{i \alpha} v_{i \beta} v_{i \gamma} v_{i \eta} W_{i}=\int_{-1}^{1} w(\mu) \mu_{\alpha} \mu_{\beta} \mu_{\gamma} \mu_{\eta} d \mu \\
=\left(\delta_{\alpha \beta} \delta_{\gamma \eta}+\delta_{\alpha \gamma} \delta_{\beta \eta}+\delta_{\alpha \eta} \delta_{\beta \gamma}\right),
\end{gathered}
$$

where, $\alpha, \beta, \gamma$, and $\eta$ are the indices for representative dimensions. These moment equations are solved for the $D_{2} Q_{8}$ LBE system, and the weights for cardinal and diagonal directions are obtained as $\frac{1}{5}$ and $\frac{1}{20}$, respectively.

\section{Numerical Examples}

\subsection{Su-Olson benchmark problem for nonequilibrium radiative transfer}

Let us consider a nonequilibrium radiative transfer problem, proposed by $\mathrm{Su}$ and Olson [10], in an initially cold (zero temperature), homogeneous, infinite, and 
Table 1: Weights for different lattices

\begin{tabular}{|c|c|c|}
\hline Lattice & Direction, $i$ & Weight, $W_{i}$ \\
\hline$D_{1} Q_{2}$ & $1-2$ & 0.50 \\
\hline$D_{2} Q_{4}$ & $1-4$ & 0.25 \\
\hline \multirow{2}{*}{$D_{2} Q_{8}$} & $1-4$ & 0.20 \\
\cline { 2 - 3 } & $5-8$ & 0.05 \\
\hline
\end{tabular}

isotropically scattering slab with an internal radiation source. An analytical solution was developed in [10] for the coupled radiation transport and material energy balance equation for a one dimensional planar geometry $x \in(-\infty, \infty)$ with negligible effect from any other mode of energy exchange. The governing one dimensional transport equations were linearized by assuming temperature-independent absorption and scattering cross-sections and assuming a cubic temperature dependence of $C_{v}$. The same system of equations with source terms, as used by [10] and described in sec. II, are used here to verify our LBM solution method.

For a 1D slab, the variable $\psi(r, \Omega, t) \equiv \psi(x, \mu, t)$ has only two directions, with direction cosines $\mu= \pm \frac{1}{\sqrt{3}}$, and governing Eqs. (7) and (8) reduce to

$$
\begin{array}{r}
\frac{1}{v} \frac{\partial \psi(x, \mu, t)}{\partial t}+\mu \frac{\partial \psi(x, \mu, t)}{\partial x}=\sigma_{a}\left[\frac{1}{2} \theta(x, t)-\psi(x, \mu, t)\right]+ \\
\sigma_{s}\left[\frac{1}{2} \int_{-1}^{1} \psi\left(x, \mu^{\prime}, t\right) d \mu^{\prime}-\psi(x, \mu, t)\right]+s(x, \mu, t)
\end{array}
$$

and

$$
\frac{1}{v} \frac{\partial \theta(x, t)}{\partial t}=\sigma_{a}\left[\int_{-1}^{1} \psi\left(x, \mu^{\prime}, t\right) d \mu^{\prime}-\theta(x, t)\right],
$$

where $x \in(-\infty, \infty)$ is the spatial variable, $\mu$ is the cosine of the radiation direction measured with respect to the $\mathrm{x}$-axis, $t \in(0, \infty)$ is the time variable, $\psi$ is the radiation intensity, $\theta$ is proportional to the fourth power of the local material 
temperature [Eq. (6)], $v$ is the radiation speed, $s$ is the radiation source, and $\sigma_{a}$ and $\sigma_{s}$ are the absorption and scattering cross sections of the material. An isotropic radiation source $s$ that is uniformly distributed in a finite space $\left[-x_{0}, x_{0}\right]$ is turned on at $t=0$ and remains active only for a finite period of time $\left[0, t_{0}\right]$.

The source term

$$
s(x, \mu, t)=\frac{1}{2} s_{x} s_{t}
$$

has direct dependence on space,

$$
s_{x}=\frac{1}{2 x_{0}}\left[H\left(x+x_{0}\right)-H\left(x-x_{0}\right)\right],
$$

and time,

$$
s_{t}=\left[H(t)-H\left(t-t_{0}\right)\right]
$$

where $H$ is a unit Heaviside function, $x_{0}$ is the length of half-domain where source is active, and $t_{0}$ is the time duration for which the source is active. The boundary conditions and initial condition are described below:

$$
\begin{gathered}
\psi_{\mu}(x=-\infty, t)=0, \\
\psi_{-\mu}(x=+\infty, t)=0, \\
\psi_{ \pm \mu}(x, t=0)=0 .
\end{gathered}
$$

For this 1D problem, the LBM numerical solution can only be obtained for a computational domain of finite size and is chosen to be of length $200 x_{0}$. In 1D there are only two angular directions which have direction cosines $\pm \frac{1}{\sqrt{3}}$, and each having weight fraction equal to 1 . In Fig. [2] a-b, the LBM solution is compared against the semi-analytic $P_{1}$ solution [11] obtained in an infinite domain. Results provided in the figure are only for the half-domain due to symmetry of the problem. Except for the limitation of numerical method to simulate infinite domain, 
Table 2: Parameter values used from Su and Olson [10]

\begin{tabular}{|c|c|}
\hline$\sigma_{a}$ & 1 \\
\hline$\sigma_{s}$ & 0 \\
\hline$v$ & 1 \\
\hline$x_{0}$ & 0.5 \\
\hline$t_{0}$ & 10 \\
\hline
\end{tabular}

all the other parameters and conditions are kept same as used in the analytical solution and are provided in Table [2] below.

The LBM results shown in Fig. [2] are in good agreement with the $P_{1}$ solution and thus verify the accuracy and applicability of the proposed method for such problems. It can be proved with the help of the following analysis that the two representative solutions of this problem (corresponding to both angular directions) can be used to retrieve the $P_{1}$ approximate form of the coupled integro-differential equations. Consider $\psi_{+}$and $\psi_{-}$to be the solutions corresponding to directions $+\frac{1}{\sqrt{3}}$ and $-\frac{1}{\sqrt{3}}$, respectively, which satisfy the Eqs. (18) and (19). Substituting $\psi_{+}$and $\psi_{-}$in Eqs. (18) and (19) and approximating the integral operation with a summation over the two directions yield

$$
\begin{array}{r}
\frac{1}{v} \frac{\partial \psi_{+}(x, t)}{\partial t}+\frac{1}{\sqrt{3}} \frac{\partial \psi_{+}(x, t)}{\partial x}=\sigma_{a}\left[\frac{1}{2} \theta(x, t)-\psi_{+}(x, t)\right]+ \\
\sigma_{s}\left[\frac{1}{2}\left(\psi_{+}(x, t)+\psi_{-}(x, t)\right)-\psi_{+}(x, t)\right]+\frac{1}{2} s_{x} s_{t}, \\
\frac{1}{v} \frac{\partial \psi_{-}(x, t)}{\partial t}-\frac{1}{\sqrt{3}} \frac{\partial \psi_{-}(x, t)}{\partial x}=\sigma_{a}\left[\frac{1}{2} \theta(x, t)-\psi_{-}(x, t)\right]+ \\
\sigma_{s}\left[\frac{1}{2}\left(\psi_{+}(x, t)+\psi_{-}(x, t)\right)-\psi_{-}(x, t)\right]+\frac{1}{2} s_{x} s_{t},
\end{array}
$$


and

$$
\frac{1}{v} \frac{\partial \theta(x, t)}{\partial t}=\sigma_{a}\left[\left(\psi_{+}(x, t)+\psi_{-}(x, t)\right)-\theta(x, t)\right] .
$$

The approximate forms of analytical expressions for the zeroth and first moments can be written as

$$
\phi_{0}(x, t)=\int_{-1}^{1} \psi\left(x, \mu^{\prime}, t\right) d \mu^{\prime}=\left[\left(\psi_{+}(x, t)+\psi_{-}(x, t)\right)\right]
$$

and

$$
\phi_{1}(x, t)=\int_{-1}^{1} \mu^{\prime} \psi\left(x, \mu^{\prime}, t\right) d \mu^{\prime}=\frac{1}{\sqrt{3}}\left(\psi_{+}(x, t)-\psi_{-}(x, t)\right) .
$$

Adding Eqs. (24) and (25) and then substituting the above moment expressions yield

$$
\frac{1}{v} \frac{\partial \phi_{0}(x, t)}{\partial t}+\frac{\partial \phi_{1}(x, t)}{\partial x}=\sigma_{a}\left[\theta(x, t)-\phi_{0}(x, t)\right]+s_{x} s_{t}
$$

Now multiplying Eqs. (24) and (25) with their respective directional cosines $+\frac{1}{\sqrt{3}}$ and $-\frac{1}{\sqrt{3}}$ and adding the resultant equations give

$$
\frac{1}{v} \frac{\partial \phi_{1}(x, t)}{\partial t}+\frac{1}{3} \frac{\partial \phi_{0}(x, t)}{\partial x}=-\left(\sigma_{a}+\sigma_{s}\right) \phi_{1}(x, t) .
$$

Equation (26) can be rewritten as

$$
\frac{1}{v} \frac{\partial \theta(x, t)}{\partial t}=\sigma_{a}\left[\phi_{0}(x, t)-\theta(x, t)\right] .
$$

Above Eqs. (29-31) are equivalent to the $P_{1}$ approximate form of the nonequilibrium radiative transport equations and can readily be solved [11]. 


\subsection{Two-dimensional nuclear checkerboard benchmark problem}

Let us consider a 2D checkerboard problem that was identified by Brunner [12] as one of the benchmarks to test and compare different radiation transport methods (diffusion, flux limited diffusion, $P_{1}, P_{7}, S_{6}$ and Implicit Monte Carlo) and their underlying approximations. In this benchmark, a $7 \mathrm{~cm} \mathrm{x} 7 \mathrm{~cm} 2 \mathrm{D}$ square region (Fig. [3]) is considered which is made of a purely-scattering bulk material ( $\sigma_{t}=$ $\left.\sigma_{s}=1 \mathrm{~cm}^{-1}\right)$ with eleven purely-absorbing $1 \mathrm{~cm} \mathrm{x} 1 \mathrm{~cm}$ checkerboards $\left(\sigma_{t}=\sigma_{a}\right.$ $=10 \mathrm{~cm}^{-1}$ ) and surrounded by vacuum on the outside of domain boundaries, while continuity of angular flux is assumed at the interfacial boundaries within the domain. There is a particle source present in the central square (colored white in Fig. [3]) of the domain which also acts as a scattering region. Furthermore, it is assumed that the particles, once absorbed, are removed from the system without heating it up; therefore, eliminating the need to model the material energy equation. In addition, the particles are assumed to travel at a constant speed of $1 \mathrm{~cm} / \mathrm{s}$ inside the domain.

The governing one-speed neutron transport equation for this benchmark problemwith assumed isotropic scattering and without coupling to the material energy equation-can be written as follows:

$$
\begin{array}{r}
\frac{1}{v} \frac{\partial \psi(r, \Omega, t)}{\partial t}+\Omega . \nabla \psi( \\
+\frac{\sigma_{s}}{4 \pi} \int_{4 \pi} \psi\left(r, \Omega^{\prime}, t\right) d \Omega^{\prime} \\
+s_{0}(r, \Omega, t),
\end{array}
$$

where $\psi(r, \Omega, t)$ is a mono-energetic (one-speed), space- and time-dependent, angular neutron flux with the direction of neutron travel characterized by the unit vector $\Omega=\frac{v}{|v|} ; v$ is the neutron speed; $s_{0}(r, \Omega, t)$ is a space- and time-dependent 
source of mono-energetic neutrons; and $\sigma_{s}$ and $\sigma_{t}=\sigma_{s}+\sigma_{a}$ are scattering (isotropic) and total cross-sections, respectively. Table [3] provides values chosen for the absorption $\sigma_{a}$ and scattering $\sigma_{s}$ cross-sections and other parameters for this benchmark problem.

This described $D_{2} Q_{8}$ scheme is used to solve the lattice problem with grid spacing $\Delta x=\Delta y=0.025 \mathrm{~cm}$. In Fig. [4], LBM simulation results for $\log _{10} \sum \psi$ are compared with the corresponding discrete ordinate method (DOM) solutions, provided by Brunner [12] for different times $(t=3.2 \mathrm{~s}$ and steady state $\mathrm{t}=20$ $\mathrm{s}$ ), after the source is turned on at $\mathrm{t}=0$. At $\mathrm{t}=3.2 \mathrm{~s}$, particles get just the right amount of time to reach the principle boundaries but not the corners of the computational domain. The two dimensional contours of total radiation intensity from both methods in Fig. [4] look similar, and are particularly in a very good agreement near the source region.

The line plots along the diagonal direction of checkerboard, shown in Fig. [5], present more clearly this agreement between LBM and DOM in the internal region. However, away from the source and near the boundaries the results from two methods don't show high degree of agreement. This can be attributed to the different number of directions in the $S_{6}$ DOM and $D_{2} Q_{8}$ LBM methodology, and due to different orientation of the discrete directions used in the numerical solutions. The aforementioned numerical differences between the two methods can be corrected by adopting the following possible corrective measures (i) Increasing the number of directions in a lattice, or (ii) by transporting particles beyond the first neighbors, or (iii) by introducing $P_{N}$ corrective terms in the LBM algorithm based on the spherical harmonics approximations conserving certain moments of the Boltzmann equation 
Table 3: Parameter values for the two dimensional nuclear checkerboard benchmark problem [12]

\begin{tabular}{|l|r|r|r|}
\hline Box & Red & Blue & White \\
\hline$\sigma_{a}\left(\mathrm{~cm}^{-1}\right)$ & 10 & 0 & 0 \\
\hline$\sigma_{s}\left(\mathrm{~cm}^{-1}\right)$ & 0 & 1 & 1 \\
\hline$\sigma_{t}\left(\mathrm{~cm}^{-1}\right)$ & 10 & 1 & 1 \\
\hline$v(\mathrm{~cm} / \mathrm{sec})$ & 1 & 1 & 1 \\
\hline$s_{0}\left(\# / \mathrm{cm}^{3}-\mathrm{sec}\right)$ & 0 & 0 & 1 \\
\hline
\end{tabular}

\section{Conclusions}

This paper extends the recently proposed LBE [1] for solving benchmark nonequilibrium radiative and neutron transport problems. Motivation behind this work is to unify the computational framework for solving highly coupled multiphysics problems in nuclear and thermal engineering. The time-dependent nonequilibrium radiative transfer problem is a classical one dimensional problem where radiative and material energy are mutually coupled. The LBE solution of this problem is shown to be equivalent to $P_{1}$ form for all times. The most common practical application of linear Boltzmann equation is for simulating neutron population in nuclear reactors. A two dimensional benchmark problem of checkerboard lattice cells with source, scattering and absorbing media is solved using $D_{2} Q_{8}$ LBE. The results closely agree with the solution from $S_{6}$ DOM, except near the boundaries. The Lattice Boltzmann method experiences similar problem of ray effects as in case of discrete ordinates method, and further work is necessary to mitigate such unphysical numerical effects. However, results so far show that LBE can be a powerful tool for solving coupled multi-physics problems which can be described by Boltzmann equation. Thus, further efforts should be directed to extend this LBE framework for higher dimensions, higher accuracy with more 
discrete directions and multi-energy levels.

\section{References}

[1] Bindra H., Patil D. V., “ Radiative or neutron transport modeling using a lattice Boltzmann equation framework ”, Physical Review E., 86, 016706, (2012)

[2] McCulloch R., Bindra H.," Coupled radiative and conjugate heat transfer in participating media using lattice Boltzmann method ", Computers \& Fluids, $124,261-269,(2016)$

[3] Briesmeister J. F., “ MCNP'TM: A General Monte Carlo N-Particle Transport Code ", LA13709M, (2000)

[4] Bowman S. M., " SCALE 6: Comprehensive Nuclear Safety Analysis Code System ", Nucl. Technol. 174(2), (2011)

[5] Evans T. M., Clarno K. T., Morel J. E.,“ A Transport Acceleration Scheme for Multigroup Discrete Ordinates with Upscattering ”, Nucl. Sci. Eng., (2010).

[6] Hursin M.," Full Core, Heterogeneous, Time Dependent Neutron Transport Calculations with the 3D Code DeCART ", University of California, Berkley, Ph.D Thesis, (2010)

[7] Marshak, R. E., “Effect of radiation on shock wave behavior ”, Physics of Fluids, 1, (1958)

[8] Pomraning G. C., “ The non-equilibrium Marshak wave problem ”, Journal of Quantitative Spectroscopy and Radiative Transfer, 21, 3, (1979) 
[9] Ganapol B. D., Pomraning G. C., “The non-equilibrium Marshak wave problem: A transport theory solution ”, Journal of Quantitative Spectroscopy and Radiative Transfer, 29, 4, (1983)

[10] Su B., Olson G. L., “ An analytical benchmark for non-equilibrium radiative transfer in an isotropically scattering medium ", Annals of Nuclear Energy, $24,13,(1997)$

[11] McClarren R. G., Holloway J. P., Brunner T. A., “Analytic P1 solutions for time-dependent, thermal radiative transfer in several geometries ", Journal of Quantitative Spectroscopy and Radiative Transfer, 109, 3, (2008)

[12] Brunner T. A., “Forms of approximate radiation transport ", Sandia Report, SAND2002-1778, Sandia National Laboratories, (2002)

[13] Wolf-Gladrow D. A.,“ Lattice-Gas Cellular Automata and Lattice Boltzmann Models - An Introduction ”, Springer, (2005)

[14] Kandhai B. D.,“ Large Scale Lattice-Boltzmann Simulations ”, Computational Methods and Applications, Thesis, Universiteit van Amsterdam, (1999) 

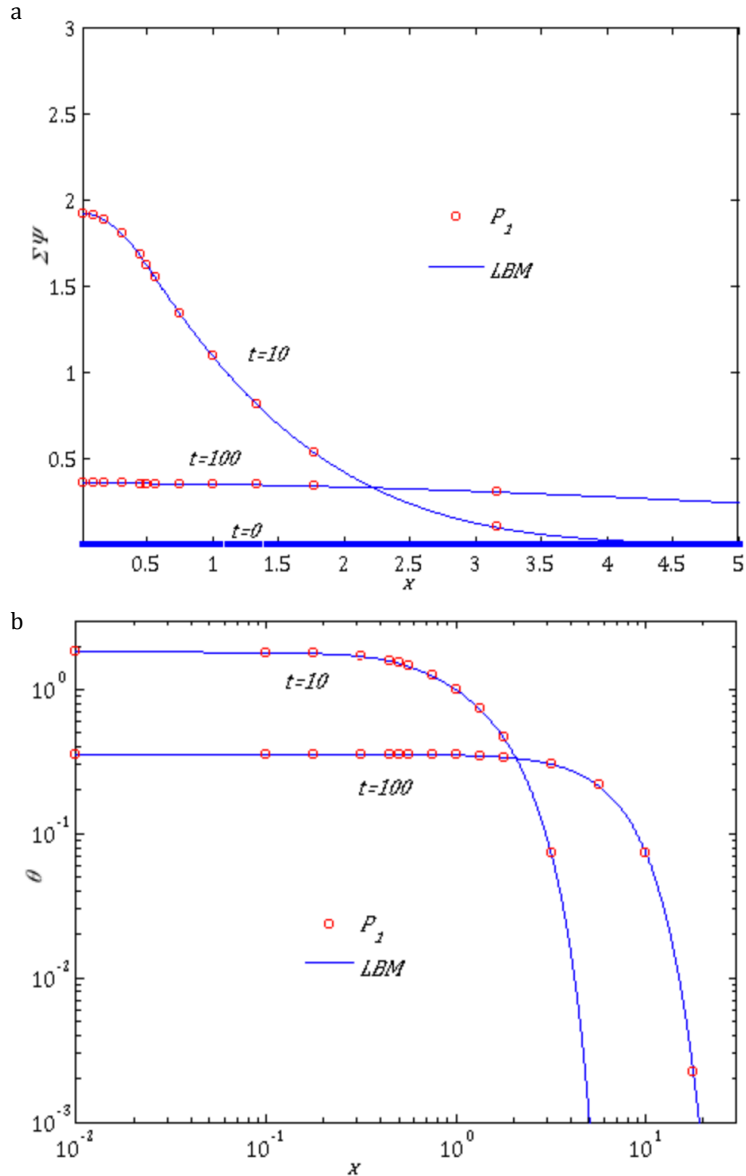

Figure 2: Solution for the Su-Olson problem with Table [2] parameters using LBM: a) Radiative Energy b) Material Energy 


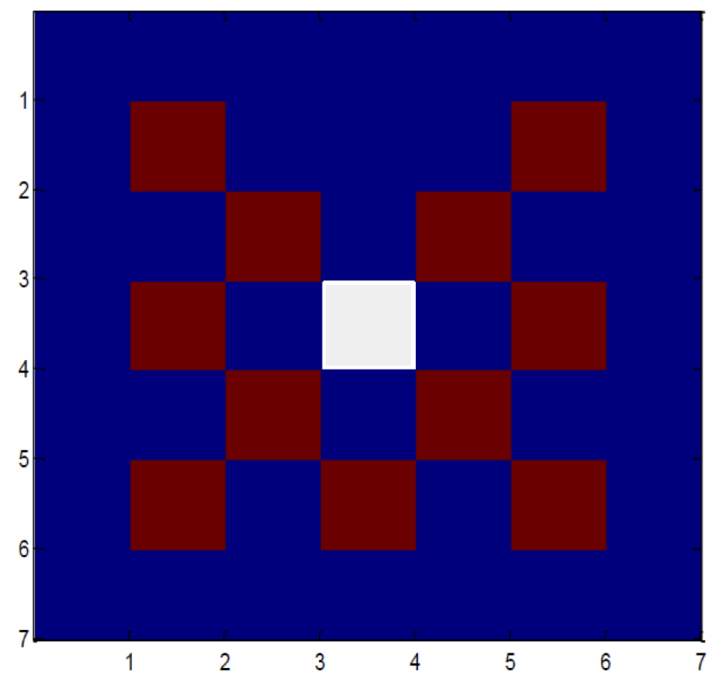

Figure 3: Two dimensional nuclear checkerboard benchmark problem. Blue and red colored regions are purely scattering and purely absorbing regions, respectively. White region in the center acts as a combined source and scattering region. 

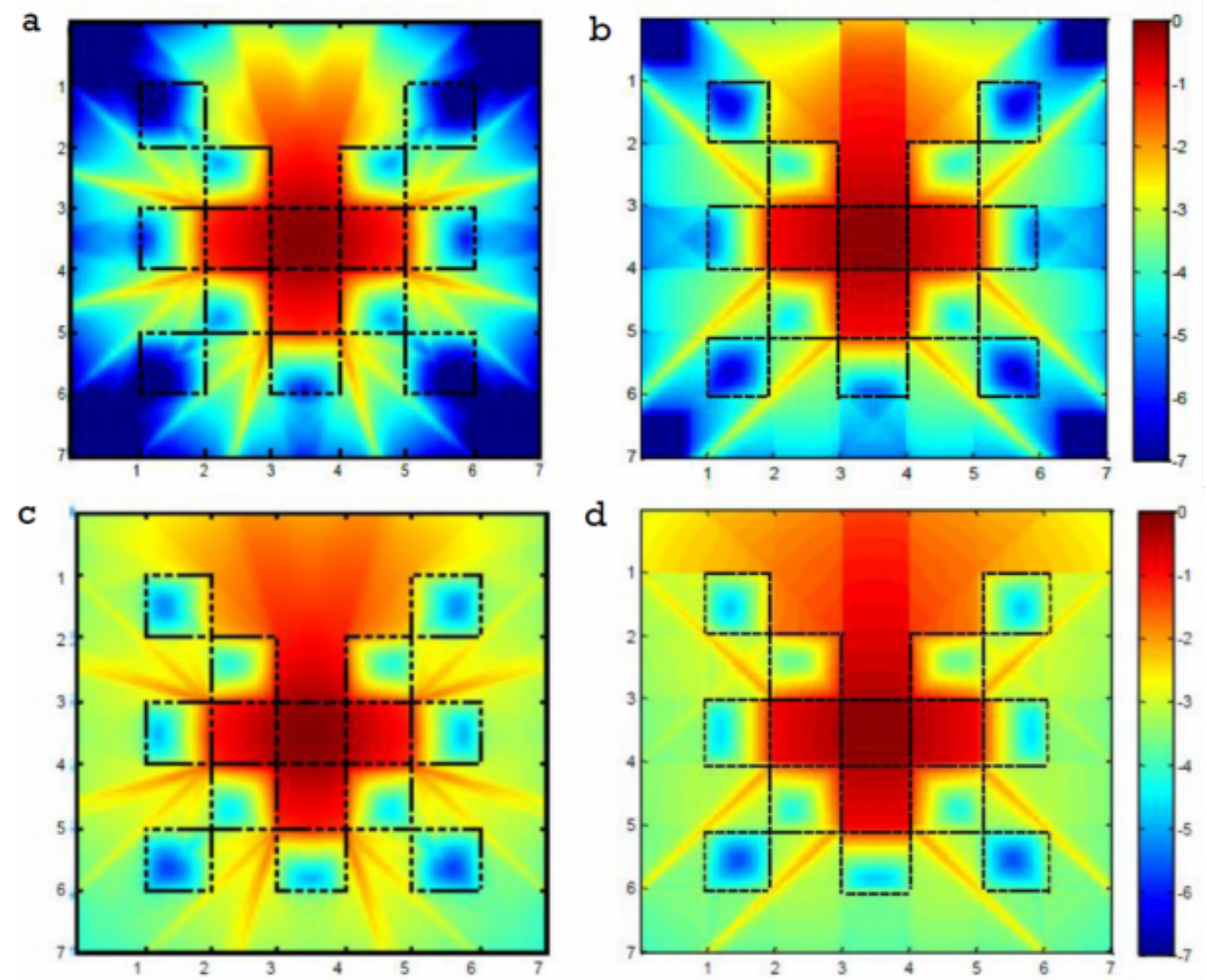

Figure 4: Nuclear checkerboard problem solution for $\log _{10} \sum \psi$ with Table [3] parameters: a) $S_{6}$ solution at $3.2 \mathrm{sec}, \mathrm{b}) \mathrm{LBM} D_{2} Q_{8}$ solution at $\left.3.2 \mathrm{sec}, \mathrm{c}\right) S_{6}$ solution at steady state, d) LBM $D_{2} Q_{8}$ solution at steady state 


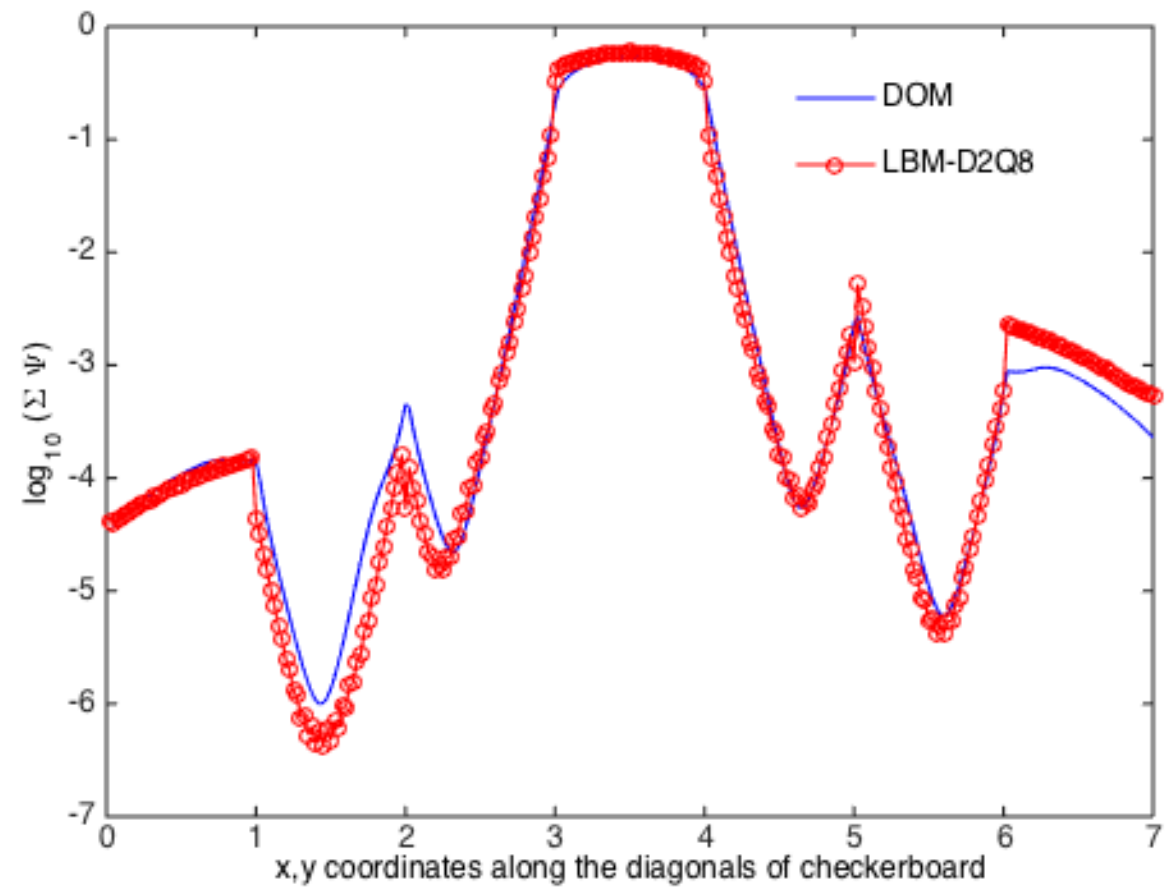

Figure 5: Line plots ( $D_{2} Q_{8}$ vs DOM $\left.S_{6}\right)$ for nuclear checkerboard problem solution 